\title{
Optimal Hedging Strategy for Risk Management on a Network
}

\author{
Tianjiao Gao, Aparna Gupta and Nalan Gulpinar
}

Dec 18th, 2012

\begin{abstract}
In this paper, we derive and assess a framework in which a firm's financial environment is an integral part of its hedging decisions. In the framework, the characteristics of firms in the network and their interconnections affect the firm's risk management strategy through impact on contract cost and efficacy of protection. We apply the model to an investment fund's decision making problem for transferring equity risk to a set of banks and obtain an a priori optimal hedging decision based on a risk-return tradeoff analysis. We find hedge costs greatly influence the fund's choice of counterparties for contract: the cost advantage of a counterparty would award it a dominant role in the magnitude of protection sought from the counterparty. In the a posteriori analysis, we evaluate the hedge efficacy in terms of the counterparties' ability to honor the hedge contract. The counter-party risk is investigated by introducing network-caused default probability and recovery rate to our model. We find that the optimality of hedging contract in the a posteriori analysis depends on the corporate objective and risk measures. Firms focusing on minimizing variance of firm value might consider a small deviation from the a priori optimal strategy; while those focussed on lowering tail risk tend to stay with the a priori optimal strategy.

Keywords: Risk management; Hedge; Network; Optimal hedging strategy
\end{abstract}




\section{Optimal Hedging Strategy for Risk Management on a Network}

\section{Introduction and Background}

Over the past decades, globalization and capital liberalization have created a more interconnected financial systems. With the increased interdependence of economic entities, network risk capturing financial interdependence becomes a hot topic among executives. Financial innovation and rapidly developing global markets provide broader space and more channels for corporations' and financial institutions' risk management. Besides, academics are also displaying great interest in corporate risk management activities. A number of studies discuss the rationale of corporate hedge behavior and develop models for hedging decision making. However, there is no paper to the best of our knowledge incorporating firm's financial environment into the analysis of optimal hedging strategies. We believe our paper is the first one to investigate corporate optimal hedging decisions under a network setting.

The problem of hedge in risk management is traditionally seen as a bilateral decision, where one firm or agent transfers risk to others by paying certain premiums, which is the direct hedging cost. The transaction, seen as a bilateral relation, is incapable of capturing broader features of the two parties involved in the transaction. However, the broader features are important to capture counter-party risk, contagion risk, information asymmetry, etc. This is particularly relevant when there is no intermediary facilitating risk transfer, such as a clearing house or an exchange. The intermediaries, for their own interest, put in place measures and processes to protect themselves and the concerned parties for these additional risks underlying the risk being transferred. Hence, in the case of 'over-the-counter' risk transfer mechanisms, the concerned parties can affect each other by the nature of the risk transfer due to their position in the relevant domain.

The relevant domain mainly depends on the nature of risks a firm is exposed to and chooses to explicitly manage. For instance, an insurance provider may choose to transfer the tail risk of the loss distribution of its liabilities to a set of reinsurers, while hedging 
the interest-rate risk and equity market risk on its asset side with some investment banks using customized over-the-counter derivatives. In this case, all the reinsurers and investment banks, as well as other firms that interact with them for the purpose of transferring risk, will form the relevant domain. These interactions can be represented as a network, where nodes are the various firms and edges between nodes capture the transfer of risk between firms as well as characteristics of transfer, such as the magnitude of risk transfer, types of risk being transferred, instruments used for risk transfer, etc. In the finance literature, looking at the hedging and risk management problems with this broader approach is gaining attention with a recognition of the importance of impact of firms' connectivity. In different segments of the finance sector, these connectivities can have catastrophic implication, and accounting for them in a firm's hedging and risk management decisions is prudent.

In this paper, we develop a framework to create a hedging strategy responsive to a firm's position in a financially inter-connected and interdependent environment. The framework is applied on a specific case to assess the impact of the network structure of a firm's financial environment on its optimal hedging decisions. In addition, we investigate the counter-party risk in the a posteriori analysis and see how the potential default events would threaten the protection. In the a priori risk-return analysis, we find that the network features work on corporate hedging decision mainly through their impact on hedge cost. Risk managers are more likely to choose their hedging partners with comparative cost advantage compared with other competitors. While counter-party risk, which cannot be priced in the a priori analysis, is considered in the a posteriori analysis, we find there is a little chance that a slight modification from the a priori optimal hedging decision is beneficial in reducing variance of firm value. If the risk manager cares more about tail risk instead of variance of firm value, staying with the a priori strategy is more likely to happen.

The contribution of this paper is that it is one of the first analysis that incorporates firms' financial environment into developing its risk management strategies. In contrast to most previous studies on corporate hedging behaviors, we investigate a firm's optimal hedging decision making with a board view on the whole network maps it faces, instead of studying a firm-to-firm contract in isolation. We believe this method is important especially in today's highly-connected financial systems and applicable to firms in various financial environment. 
This paper contributes to risk managers by providing guidance in selecting counterparties for risk management. Besides, as a pioneer study that emphasizes financial networks in hedging strategy, it can be taken as an initial point for future research in this area. All kinds of risk, a large variety of hedging instruments, large, complex and sophisticated financial networks promise many future explorations.

The rest of the paper is organized as follows: in Section 2, we provide an overview of the background literature relevant to risk management framework developed in this paper; then we present our model in support of the risk management framework and elucidate it through a specific example in Section 3. In Section 4, we implement the model and utilize it to determine an optimal hedging strategy for the example presented in Section 3. In Section 5 in an a posteriori analysis, counter-party risk is considered in the model and contract efficacy is analyzed under different settings of a posteriori network features. The last section is the conclusion.

\section{Literature Review}

In this section, we give a brief review of previous works that relate to this paper. The first part is the literature review of risk management, in which the three basic questions "who, why and how" in corporate hedging practices are discussed. We also introduce some papers that study corporate behaviors and economic phenomena in a network framework in the second part.

\subsection{Hedging for Risk Management}

According to the Modigliani-Miller theorems (1958 [1]), shareholders can diversify their portfolio risks, so that firms' value would not be affected by the "total" risk (Nocco and Stulz, 2006 [2]). Nevertheless, due to capital market imperfection in the real world, firm's value can be increased by managing the firm's risk (Bartram, 2002 [3]). The underlying reason why risk management does not provide benefit under Modigliani-Miller assumptions is because of absence of "imperfections" in the capital markets. Taxes and bankruptcy costs, restrictions on borrowing or lending, information asymmetry, are examples of "imperfections" 
that exist in the real world that justify corporate risk management. According to previous literature, reduction of tax liability, mitigation of under-investment problem and managerial risk aversion are three main motivations for risk management.

Smith and Stulz (1985 [4]) claim that hedging, by reducing volatility of taxable income, lowers the expected corporate tax liability and increases the expected post-tax value of the firm, if the firm faces a convex tax regime with progressively increasing marginal tax rates. Nance et al. (1993 [5]) provide empirical and theoretical support for Smith and Stulz's (1985 [4]) claim that firms facing an increasing marginal tax rate tend to hedge more.

Risk management can be viewed as a substitute for the need to raise equity capital (Froot, Scharfstein and Stein 1993 [6], Stulz 1996 [7], Nocco and Stulz 2006 [2]). By lowering the volatility of firm value, a firm can satisfy its equity capital requirement to cushion its operating risks, and at the same time lower the probability of occurrence of financial distress. Through reduction of "investment distortions" and protection of net cash flows , risk management can mitigate corporate under-investment problem and lower the cost of bankruptcy, thereby increasing firm's value (Stulz 1996 [7], Froot, Scharfstein and Stein $1993[6])$.

Managerial risk aversion is another rationale given for firm's participating in explicit risk management (Mayers and Smith 1982 [8], Smith and Stulz 1985 [4]). Through empirical analysis of the North American gold mining industry, Tufano (1996 [9]) suggests that risk management policies would be affected by managerial risk aversion. The reason for hedging lies in the high degree of non-diversification in the manager's portfolio. Nevertheless, some of the hedging strategies are implemented purely for managers' interest, which could be harmful for the shareholders. Compensation scheme is one way to adjust managers' incentives to mitigate the conflict of interest between managers and shareholders. Bartram (2002 [3]) also claimed that risk management is a "value-creating" activity, because it can eliminate the "noise" in the evaluation of managers' performance, which enhances the effectiveness of the incentives in the compensation scheme.

After answering why firms should hedge, the second question that arises is how a firm should hedge. The types and extents of risk exposures are the primary determinants of firm's intensity and magnitude of hedging activities. Based on the corporation value-maximization 
assumption, Smith and Stulz (1985 [4]) examine how tax, contracting costs and the impact on investment decision affect hedging strategies and explain the variety of hedging behaviors observed among different firms. Company ownership structure and firm size also appear to impact firm hedging behavior (Smith 1995 [10], Stulz 1996 [7], Nocco and Stulz 2006 [2]).

Variance minimization is widely considered as the goal of a firm's hedging strategies, because the reduction of volatility of firm value is prevailingly believed to be the primary objective of risk management. The Value at Risk, or VaR, which measures the probability of lower tail outcomes, is widely used by financial institutions and industrial firms to monitor

their risk exposures. Ahn et al. (1999 [11]) provide a formal analysis of optimal hedging strategy using options in a simplified framework by minimizing a company's VaR. Cash flow simulations for assessing default probabilities, as another risk measure, is proposed by Stulz (1996 [7]) based on his argument of the different objectives of risk management. He suggests that to avoid "lower-tail outcomes" while preserving upside potential should be the primary aim of hedging. In the same paper, Stulz supports selective hedging strategy rather than full-covered hedging strategy by arguing that firms with "comparative advantage in bearing certain financial market risks" should take on those risks.

\subsection{Network Literature Review}

The study of networks has been an area of active study in fields of computer science, applied mathematics and operations research, and in recent years there has been an increasing interest in the social science and business fields. Networks help describe the relations and interactions between various entities connected in a network, analyze individual optimal strategy for participation in a network, allow researchers to better understand and investigate behaviors of firms. With the increased interdependence and interaction between economic agents, not only people in business, but regulatory entities also begin to pay greater attention to network issues. Researcher have applied networking theory to issues in corporate cash flow management, global tax liability of multinational corporations, multinational payment netting, capital budgeting problems and portfolio selection under uncertainty (Rutenberg 1970 [12], Srinivasan and Kim 1986 [13], Mulvey and Vladimirou 1992 [14]).

According to previous literature, risk sharing or risk transferring is one of the primary 
incentives for economic entities to develop linkages with one another. For instance, by holding interregional claims on each other, banks provide insurance against liquidity preferences shocks (Allen and Gale 2000 [15]). Corporations and financial institutions use various financial vehicles to transfer risks to others by creating networked channels of risk propagation. Inclusion of a network structure facilitates the definition of the appropriate optimization model, which provides a useful tool for studying organization behaviors towards currency risk (Jones $2000[16])$. Jones (2000 [16]) formulates a framework for networked foreign exchange trading model. By graphically describing the cash flows in networks, he generalizes the model into the application of arbitrage, hedging and speculation of currency risk. Increased dependence in supplier networks makes a firm vulnerable to uncertainty and counter-party risk, which is an important topic of research in supplier networks (Hallikas et al. 2004 [17]). From the prospect of individual economic agents, Fafchamps and Gubert (2007 [18]) investigate whether risk-sharing networks are formed to maximize mutual gains from pooling income risk.

On the other hand, practitioners and researchers have not forgotten the dark side of networks. Financial contagion, the inevitable byproduct of economic linkages, has been received increasing attention over the past decade due to frequent recurrence of banking crises. Small exogenous shocks, initially from certain financial institutions or certain economic sectors, spread by contagion through links to their "neighbors", and finally impact the whole economy. In Allen and Gale's paper (2000 [15]), the possibility of financial contagion, which is initially triggered by adverse news of insolvency of banking institutions, were greatly determined by the completeness of structure of interregional claims. Network models are analyzed by other researchers for financial system stability, banking regulation, structural interdependencies among the debtors within a credit portfolio (Thurner,Hanel and Pichler 2003 [19], Boss et al. 2004 [20], Egloff, Leippold and Vanini 2007 [21], Nier et al. 2007 [22]).

In this paper, we adopt a similar approach as the financial contagion papers. A firm's financial environment is modeled as a network, consisting of the reference firm and its counterparties as nodes, interlinked through hedge contracts. We investigate how a firm's network impacts its transaction costs and therefore optimal hedging choice as well as contract performance. Previous study points out that structured products are in general mispriced 
and there is price deviation from theoretical estimation (Wasserfallen and Schenk 1996 [23], Stoimenov and Wilkens 2005 [24], Fusai and Zanotti 2011 [25]). Following what they find, we assume there are spread adjustments from network that make real prices of financial instruments deviate from their theoretical prices.

We believe one of the most important explanations of financial instruments' price deviation is due to contract demands, counter party features, searching process and bargaining power of both contract parties. According to previous literature, searching process and bargaining power have been admitted to play an important role on asset prices in over-thecounter markets (Duffie, Gârleanu and Pedersen 2005 [26], 2007 [27]). It is argued that a higher spread is associated with the difficulty to find counterparties and less bargaining power of counterparties. Other papers argue that information disclosure and accounting transparency improve firms' bargaining power and lower transaction costs (Duffie and Lando 2001 [28], Yu 2005 [29]). Lang and Lundholm (1993 [30]) find that the accounting transparency is positively associated with firm size and performance, which further indicates firm size has impact on bargaining process and therefore spreads.

Given above evidence, we argue that a firm's network features, including link properties and node properties in the bilateral transaction, impact bargaining process as well as how to make optimal decisions. Extending the previous literature in risk management, which mainly focuses on the rationale and determinants of corporate hedge, we derive a networkbased model to capture the optimal corporate hedging strategies in both an a priori analysis and an a posteriori analysis.

\section{The Model}

In our model development for risk management on a network, we utilize the network concepts to describe risk transferring between corporations or financial institutions, and its implications to capture corporate hedging behaviors. In this section, we will describe the procedure of network-based model development in detail and apply the model in a specific example of an equity hedge fund. 


\subsection{Model Description}

In the model, $\left\{\tilde{R}_{i} ; i=1 \ldots N\right\}$ represent $N$ risk factors a firm identifies as its risk exposures to hedge. These risk factors could be arising from market, credit, operational, business, legal, reputation risks, however our focus in this article will be on the first three types of risks. We will further classify market risk into its major components of equity, interest rates, commodity prices and currency risks. There are $M$ potential counterparties selected as risk takers. For complete generality, lets assume each firm is capable of providing a risk transfer option for each risk type. Later this can be modified by imposing constraints on the extent of hedge a firm can offer for a specific risk type. The hedging option for risk type $i$ that the reference firm seeks from firm $j$ is denoted by $x_{i j}$, expressed as a fraction of the total exposure of risk factor $i$. This transfer happens at a premium, incurred by the reference firm towards the firm $j$. It is a hedging cost to the reference firm for instituting the risk transfers.

The cost of hedge applicable to a risk, $h_{i j}$, not only is a function of the type of risk and the extent of risk transfer, but also the position of the reference firm and the firm $j$ in the network. Therefore, to quantify the cost of hedge, it is important to incorporate all the firms in the network in ways that sufficiently capture their financial environment. In order to describe the financial environment, instead of growing the network to incorporate all the firms the $M$ firms transact with, which will potentially become a prohibitively large network to study, we incorporate the essence of this larger network in the nodes and links properties of the network with $M+1$ firms. Given the structure of the network, the hedging cost, $h_{i j}$, is modeled as a function of extent of risk exposure, the magnitude of risk transferring, and several important factors that implicitly impact contract costs according to previous literature.

Bargaining power has been indicated an important role in contract setting up (Duffie, Gârleanu and Pedersen 2005 [26], 2007 [27]). When counterparties meet, contract prices are set through a bargaining process to reflect both sides' features. Firms with bigger sizes are usually believed to take advantages in the bargaining process, which means the the relative size between counterparties matters. Also, information asymmetry may cause higher spreads since accounting intransparency endues longer bargaining time and greater transaction costs 
. These has been empirically tested by Duffie and Lando (2001 [28]) and Yu (2005 [29]). In addition, previous researchers find that banks prefer to provide discount to reward clients with longer relationship with them. Thus we believe longevity of business relationship greatly impact contract price. Due to above argument, we add several network features into our model, such as, 1. credit rating of reference firm and risk taker $j$ in the network, 2. age of the link, i.e. longevity of relationship between the reference and the firm j, 3. transparency of reference firm's operations and that of firm $j$ 's operations, 4. relative size of firms, etc.

Given hedge cost of each risk type on each firm described above, the total cost is the summation of them, as follows,

$$
H=\sum_{i=1}^{N} \sum_{j=1}^{M} h_{i j}\left(z_{i} p\left(x_{i j}\right) ; f_{1}, f_{2}, f_{3}, f_{4}\right)
$$

where $p($.$) is the theoretical price of the hedge and f_{i}$ refers to the factors that determine the cost of hedge for each risk type and transacting firms' characteristics. Given the extent of exposure of the reference firm to each risk type, denoted by $\mathbf{Z}=\left[z_{i}\right]_{\{i=1: N\}}$, the payoff to the firm from these risk exposures is a function of the risks, $\tilde{R}_{i}$, and hedges put in place, $\sum_{j=1}^{M} x_{i j}$, for that risk. In order to capture the interaction of the risk factors and the hedges to determine the total pay-off at the planning horizon, we incorporate $\tilde{\mathbf{R}}=\left[\tilde{R}_{i}\right]$ and $\mathbf{X}=\left[x_{i j}\right]_{\{i=1: N, j=1: M\}}$ into the pay-off function,

$$
\tilde{Y}=Y(\tilde{\mathbf{R}}, \mathbf{X})
$$

We presently consider a single-period problem, where the reference firm's risk exposures are realized at $t=1$ and the hedge decisions are made and cost of hedge incurred at $t=0$. The hedging decision includes three main questions, what, how and who. Another important part in reference firm's decision making of risk management is to choose the goal of risk reduction, in other words, the appropriate risk measure. In past literature, variance of firm value is generally used among academics; and tail risk measures, such as Value-at-Risk (VaR) and Conditional Value-at-Risk (CVaR), are also widely used among executives. We will use these measures in our a posteriori analysis to evaluate the performance of established hedge 
contract. Before that, we conduct a risk-return tradeoff analysis in the a priori analysis, which incorporates a firm's risk alleviating objective and its value preserving goal in our objective function.

We first generate the firm present value by the discounted firm pay-off if no hedge contract is established. Combining the model developed thus far, we assess the value of the firm at $t=0$ as following,

$$
\tilde{V}_{n o-h e d g e}=\frac{\tilde{Y}_{n o-h e d g e}}{1+d}
$$

where $d$ is the cost of capital for the reference firm. If the firm chooses to hedge, the hedge cost is deducted from the discounted firm pay-off to assess firm value,

$$
\tilde{V}_{\text {hedge }}=\frac{\tilde{Y}_{\text {hedge }}}{1+d}-H
$$

The risk exposure to the firm's value is $\sigma_{n o-h e d g e}$ in no-hedge situation and $\sigma_{\text {hedge }}$ in hedge situation. Hedging induced premium payment is a cost burden on the firm's value, controlling which is an added concern for risk managers. To make this trade-off the formalized objective of the problem, we consider an objective function, $\sigma_{\text {hedge }}^{2}+\left(E\left[\tilde{V}_{\text {no-hedge }}\right]-E\left[\tilde{V}_{\text {hedge }}\right]\right)$. To reach an optimal hedging strategy, the firm wants to maximize the benefit of hedge, which is to minimize the risk exposure after hedging, as well as to minimize the cost of hedge on the firm value $\left(E\left[\tilde{V}_{n o-h e d g e}\right]-E\left[\tilde{V}_{\text {hedge }}\right]\right)$. The decision for the firm is to choose the extent of hedges on each risk type among counterparties, $x_{i j}$ with the $M$ firms in such a way that the objective function is minimized. Therefore, the optimization problem is defined as,

$$
\begin{array}{cl}
\min _{x_{i j}} & \sigma_{\text {hedge }}^{2}+\left(E\left[\tilde{V}_{\text {no-hedge }}\right]-E\left[\tilde{V}_{\text {hedge }}\right]\right), \\
\text { such that } & H \leq \rho_{h}, \\
& 0 \leq \sum_{j=1}^{M} x_{i j} \leq 1 \\
& \alpha_{i j} \leq x_{i j} \leq \beta_{i j},
\end{array}
$$

where $\rho_{h}$ is the upper bound on the cost of hedge and $\alpha_{i j}, \beta_{i j}$ are the bounds on the extent 
of hedge the reference firm can institute for each risk type with each firm in the network. These bounds must be constructed to constrain the risk transfer decision based on whether, and to what extent, the reference firm should transfer risk $i$ to the firm $\mathrm{j}$. Should the solution reach the lower bound in eqn (8), this would be an indication that the reference firm is only willing to cover the minimum amount of its risk, which might be due to high hedge costs; while at upper bound means maximum coverage is the best strategy. We will implement the model for a specific example in the following subsection and discuss the details of model utilization.

\subsection{An Equity Hedge Fund Example}

We now develop a specific example to elucidate the network structure model developed thus far. A reference firm engaged in equity investment, essentially an equity hedge fund, is considering to build several risk transferring contracts with a set of investment banks. Refer to schematic in Figure 1. To keep the analysis easily demonstrated, let's consider the fund invests in 3 stocks, therefore the primary risk exposure is the equity risk. The fund owns $z_{i}$ number of shares of each stock, and constructs over-the-counter hedges for the 3 equity risk factors, $\tilde{\mathbf{R}}=\left[\tilde{R}_{i}\right]_{\{i=1: 3\}}$, and transacts with 2 investment banks to institute these hedges, $\left[x_{i j}\right]_{\{i=1: 3, j=1: 2\}}$. The initial stock price at $t=0$ is $\left[S_{i}(0)\right]_{\{i=1: 3\}}$ and the one-period return of

the stock is $\tilde{\mathbf{R}}=\left[\tilde{R}_{i}\right]_{\{i=1: 3\}}$, hence an unhedged pay-off from equity investment would be, $\sum_{i=1}^{3} z_{i} S_{i}(0) \tilde{R}_{i}$.

When the hedge, $\mathbf{X}=\left[x_{i j}\right]_{\{i=1: 3, j=1: 2\}}$, is put in place, we need to determine the hedged pay-off and the hedge cost. In order to describe the hedge cost for the risk transfers, we need to develop a description of each firm node $\left(\mathbf{F}_{\mathbf{i}}\right)$ and link $\left(\mathbf{L}_{\mathbf{i j}}\right)$ of the network as Figure 1. The description of the nodes include connectivity $\left(f_{1}\right)$, riskiness $\left(f_{2}\right)$, size of the firm $\left(f_{3}\right)$, and transparency of the firm's operations $\left(f_{4}\right)$, and the description of the links include age of the link $\left(f_{5}\right)$ and ease of link monitoring and enforcement $\left(f_{6}\right)$. We will describe the details of quantification of these characteristics in the following as well as Appendix A.

Some of the network features represent episodic occurrence of events and implication to the hedge. Therefore, in the a priori risk-return analysis, several network features are accounted for in spread adjustments. These include riskiness $\left(f_{2}\right)$, size of the firm $\left(f_{3}\right)$, 
Figure 1: The Reference Firm (Ref) transacts with two banks, 1 and 2. The links and nodes have properties in the network that dictate the hedge costs.

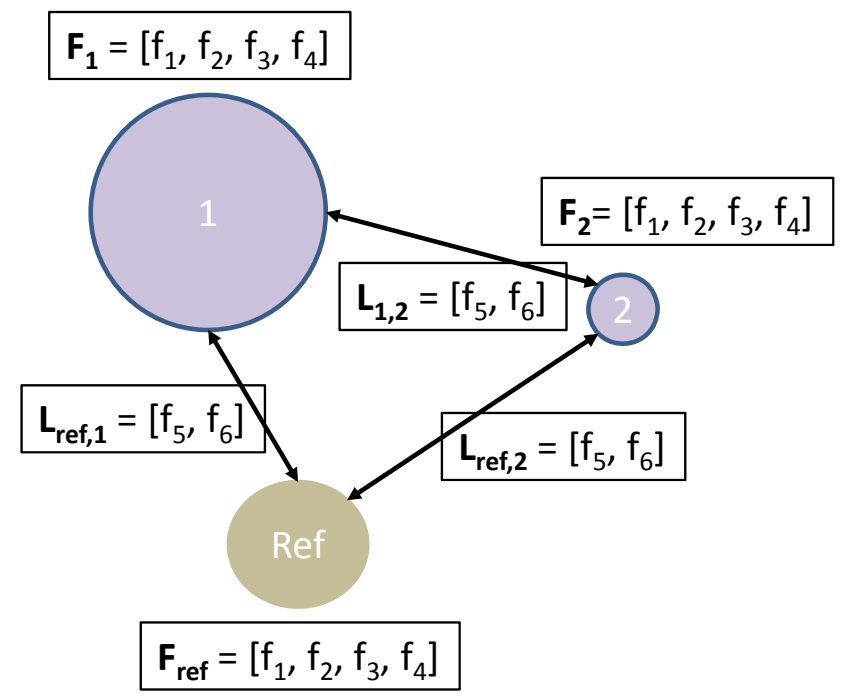

transparency of the firm's operations $\left(f_{4}\right)$, and age of the link $\left(f_{5}\right)$. However, there are several other network features which cannot be priced before contract establishment, such as connectivity $\left(f_{1}\right)$ and ease of link monitoring and enforcement $\left(f_{6}\right)$. These features will greatly impact a contract's efficacy in the post-contract establishment period, especially in the event of default. We delegate them for inclusion in our a posteriori analysis.

In the a priori analysis, the cost of hedge can be constructed by following equation,

$$
H=\sum_{i=1}^{3} \sum_{j=1}^{2} h_{i j}\left(p\left(z_{i} x_{i j}\right) ; \mathbf{F}_{\mathbf{j}}, \mathbf{L}_{\mathbf{r e f}, \mathbf{j}}\right)
$$

We consider each factor to result in a spread (or a discount) adjustment to the theoretically determined hedge instrument price $p($.$) , given by \pm s\left(f_{i}\right)$, where the sign indicates whether this adjustment is a mark-up or down. For the individual factor spread model, each factor $\left(f_{i}\right)$ needs to be mapped to its impact on marking the cost of hedge up or down per unit hedge instituted, and for their combined impact we have,

$$
h_{i j}\left(p\left(z_{i} x_{i j}\right) ; \mathbf{F}_{\mathbf{j}}, \mathbf{L}_{\mathbf{r e f}, \mathbf{j}}\right)=p\left(z_{i} x_{i j}\right)\left(\prod_{k \in \mathbf{F}_{\mathbf{j}}}\left(1+s\left(f_{k}\right)\right)\right)\left(\prod_{k \in \mathbf{L}_{\mathbf{r e f}, \mathbf{j}}}\left(1+s\left(f_{k}\right)\right)\right) .
$$


For continued simplicity in the model development, if we assume that all the hedges are vanilla European put options with strike prices $K_{i}<S_{i}(0)$, the number of options purchased to cover the long position of stock i would be $\sum_{j=1}^{2} x_{i j} z_{i}$, and the pay-off of stock i's position will be $S_{i}(0) \tilde{R}_{i}+\left(K_{i}-S_{i}(0) \tilde{R}_{i}\right)_{+}$. Therefore, the payoff at contract maturity $t=1$ is,

$$
\tilde{Y}_{\text {hedge }}=\sum_{i=1}^{3}\left[\sum_{j=1}^{2} z_{i} x_{i j}\left(S_{i}(0) \tilde{R}_{i}+\left(K_{i}-S_{i}(0) \tilde{R}_{i}\right)_{+}\right)+z_{i}\left(1-\sum_{j=1}^{2} x_{i j}\right) S_{i}(0) \tilde{R}_{i}\right]
$$

The value of firm is given by present value of pay-off minus hedging contract cost $(H)$,

$$
\tilde{V}_{\text {hedge }}=\frac{\sum_{i=1}^{3}\left[\sum_{j=1}^{2} z_{i} x_{i j}\left(S_{i}(0) \tilde{R}_{i}+\left(K_{i}-S_{i}(0) \tilde{R}_{i}\right)_{+}\right)+z_{i}\left(1-\sum_{j=1}^{2} x_{i j}\right) S_{i}(0) \tilde{R}_{i}\right]}{1+d}-H
$$

where $d$ is the cost of capital for the hedge fund. The optimization problem in eqn (5)-(8) will be stated in terms of $E\left[\tilde{V}_{\text {hedge }}\right]$ and bounds on $x_{i j}$. In this example, these bounds are set at 0 for $\alpha_{i j}$ and 1 for $\beta_{i j}$.

In the next section, we will describe the setting of parameters in this example in detail and analyze the simulation results. Through the a priori analysis, we obtain a general idea of how managers select optimal hedging strategy based on the risk-return tradeoff. However, hedging is not the executives' sole goal, which means the reference firm needs to still keep an eye on the efficacy of the established contracts before they mature. Certain characteristics of the transacting firm can result in cost-effective attractive hedge to not yield in a perfect risk transfer as per contract. Due to the characteristics of counterparties, the reference firm may be exposing itself to additional risk, such as counter-party risk. We capture these primarily through firm's connectivity $\left(f_{1}\right)$, describing the possibility of contagion spread through exogenous channels, and ease of link monitoring and enforcement $\left(f_{6}\right)$, describing legal infrastructure's impact on recovery rate in a default event. Those would be conducted in our a posteriori analysis in Section 5. 


\section{Data, Model Calibration and Analysis}

In the previous section, we develop a specific example of a fund investing in three stocks to describe our network model. To hedge against downturns in the stock market, the firm utilizes derivatives based on the performance of stocks to cover its risk exposure. The primary objective of a firm's hedge strategy is to reduce stock return volatility, at the same time to preserve firm value and deliver positive returns under all market conditions. In this section, we first give some descriptions and justifications of parameter value settings for the specific example. Then we conduct a model calibration and analyze the results in an a priori analysis.

\subsection{Parameter Description of the Model}

The properties of the three stocks owned by the hedge fund play an essential role in the hedging strategies. To reach the objective of consistency of returns and capital preservation, reference firm would give the primary consideration on factors impacting stock price, such as drift, volatility, correlation, and strike price and maturity of options. To simulate stock price movement, we set the three stocks as followings: stock 1 has a $10 \%$ annual drift rate and $15.73 \%$ volatility; stock 2 has a $6 \%$ annual drift rate and $8.15 \%$ volatility, both of which are lower than those of stock 1; stock 3 has the highest annual drift rate $18 \%$ and $31.05 \%$ volatility, which brings much equity risk to the fund. The fund's shareholdings of the three stocks are 3000, 1000 and 2000, respectively. The stock prices are assumed to follow Brownian motion and the stock prices are generated by Monte Carlo simulation. We also assume hedge tools for three stocks are one year vanilla European put options with strike price set at $90 \%$ of the initial price of the corresponding stock. The put option price is computed by the Black-Scholes formula. The amount of the put options purchased, or hedging fraction, is the decision made to balance the level of risk exposure faced by the fund and costs involved in hedging.

As model description in Section 3, we incorporate four network features in the evaluation of hedge cost. To clarify how to quantify the spread adjustment to hedge cost due to each node or linkage feature, we describe our procedures and provide justifications in this section. In Appendix A, more details of how to quantify these network features are displayed. 
Credit rating, based on the history of borrowing and repayment and the availability of assets and extent of liabilities, would be reflected through spread adjustment on hedge cost. A lower credit rating indicates lower credit worthiness of the firm, which poses as a counterparty risk and is accounted for by a mark-down on hedge cost to make the deal more attractive to the reference firm. We identify riskiness $\left(f_{2}\right)$ factor into three groups by credit rating, which is mapped to a decreasing function of spread adjustment. There is no spread adjustment for the highest rating group ([AAA/AA]), and a $-2 \%$ impact for lowest rating group ([BB and Below $]$ ). The lowest rated firms need to attract counterparties by offering lower price of hedge due to relatively low credit worthiness. This is equivalent to other linear spread adjustments we can consider, since we are primarily interested in the relative impact of these factors, the absolute values are of less significance. Riskiness is also been applied in the a posteriori analysis as indicators of theoretical default probability and recovery rate. We will discuss this in Section 5.

Size of a firm $\left(f_{3}\right)$ can impact the bargaining power of two firms in the contract establishment and to some extent, reflect the quality of the contract. Since this impact is mainly from the relative firm size point of view, we consider the size difference between the risk-taking firm and the risk-transferring (reference) firm. Four levels of size (Small, Medium, Large and Very Large) are set for firms in the network. The differential of firm size is mapped to an increasing function of spread adjustment. Compared with the reference firm, a relatively larger counterparty is more likely to have a stronger bargaining power, and tends to command a higher spread adjustment on the hedge price, therefore a positive and increasing spread adjustment $(1 \%, 2 \%, 3 \%)$ is set for a relatively larger counterparty. A smaller counterparty, compared with the reference firm is more likely to accept prices asked by the reference firm, thus a zero spread adjustment is set it.

Transparency of Firm Operations $\left(f_{4}\right)$ is considered in our model since informational asymmetry problem due to lack of transparency increases the hedge cost. This cost depends on the transparency levels of both parties in a bilateral transaction. We create four levels of transparency (Very Opaque, Opaque, Transparent and Very Transparent) and quantify them by increasing values $(0,1,2,3)$. Sum of transparency levels of two counterparties is mapped to the spread adjustment as a decreasing function. No impact $(0 \%)$ on spread adjustment 
occurs when the sum of transparency levels is large, i.e., as both firms tend to get more transparent, and vice versa $(1 \%, 2 \%)$.

In addition, we add a link property, Age of Link $\left(f_{5}\right)$, in our a priori analysis. Since the business relationship between two firms implies possibility of volume discounts, as well as the counterparties' information from transactional history. We consider four levels of link age (First Time, Short Period, Medium, and Long Term) and model the spread as a decreasing function of it. The first time transaction would raise the spread adjustment by $1 \%$ and medium and longer period would lower the spread by $1 \%$.

After giving a general idea of quantification of network features, we turn to the specific example displayed in Section 3. The three firms in the network are given different network characteristics, which would greatly impact the reference firm's hedging decision. The reference firm is set to be of a small size $\left(f_{3}\right)$ and rather opaque operation $\left(f_{4}\right)$. It would consider the corresponding characteristics of counterparies to evaluate the benefit and cost of hedging strategies. The two counterparties, as our main objective of study, can be distinguished by their specific network features.

The investment bank 1 is rated $[\mathrm{A} / \mathrm{BBB}]$ in the second level of credit rating group, which reduces the spread by $1 \%$ due to the relatively high counter-party risk. The large size of it strengthens the bank's bargaining power in the hedging transaction, increasing spread by $2 \%$; the opaque firm operation, considered together with opaque reference firm operation, raises the spread by $2 \%$. The age of relations between banks and reference firm are also counted in the risk premium evaluation. Since there is no transaction history between bank 1 and the reference firm, the spread is increased by $1 \%$.

To distinguish from bank 1 , the investment bank 2 is set to be a better hedge partner with several advantages. It has the same credit rating level $[\mathrm{A} / \mathrm{BBB}]$ as bank 1 , which reduces spread by $1 \%$. It is a small size investment bank, which is also the case for the reference firm, and transparent firm operation, together with the transparent reference firm operations, have no impact on spreads. Bank 2 already has some transactions with the reference firm before, so that this medium period relationship lowers the premium by $1 \%$ because of less information asymmetry issues as well as possible discount. Over all, considering the network impact on hedging cost, we find bank 2 has a hedging price advantage compared with its 
competitor, bank 1.

\subsection{An A Priori Model Analysis}

We compare the investment fund's optimal hedging strategies under two settings, one with the network structure and another without it. Our expectation is that the corporate decision on risk transaction would be greatly impacted by its network environment, since features of counterparties affect the costs and efficacy of contracts.

Using the parameter settings from the previous sections, we simulate the network hedging model and generate equity risk factors $\tilde{\mathbf{R}}=\left[\tilde{R}_{i}\right]_{\{i=1: 3\}}$ corresponding to three stocks owned by the fund based on $10^{6}$ replicates of simulation. $\tilde{R}_{i}$ is one-period return of stock i, which is not resampled in each iteration. To search for global minimum solution, the initial value given to each hedge fraction $\left[x_{i j}\right]_{\{i=1: 3, j=1: 2\}}$ is $50 \%$ per bank for each stock. The constraints on hedge fraction are eqn (6)-(8). The upper bound of hedge cost $\rho_{h}$ is set at $10^{10}$, which makes it irrelevant for the present moment. We use the Global Optimization Tools in MATLAB to calculate global minimum in two settings of the model, one with network adjustment on risk premiums, and another without. The simulation results are provided in Table 1.

Table 1: Optimal hedging strategies of the investment fund in different network settings

\begin{tabular}{|l|r|r|}
\hline \multicolumn{2}{|l|}{ Panel A. Model without network setting } & \multicolumn{2}{l|}{ Bank 2 } \\
\hline Hedging fraction by each bank & Bank 1 & $50 \%$ \\
\hline stock 1 & $50 \%$ & $50 \%$ \\
\hline stock 2 & $50 \%$ & $50 \%$ \\
\hline stock 3 & $50 \%$ & \\
\hline & & \\
\hline Panel B. Model with network setting & & \multicolumn{2}{|c|}{} \\
\hline Hedging fraction by each bank & Bank 1 & \multicolumn{2}{|c|}{ Bank 2 } \\
\hline stock 1 & $0 \%$ & $100 \%$ \\
\hline stock 2 & $0 \%$ & $100 \%$ \\
\hline stock 3 & $0 \%$ & $100 \%$ \\
\hline
\end{tabular}

First, we look at the total risk coverage ratios in two settings in Table 1 . We notice that the summation of percentage of each equity risk covered by the two transacting banks are 100\% in both Panel A and Panel B, which suggests the investment bank fully covers its risk exposures in stock market to obtain stable returns if there is no restriction on hedge cost. 
Second, we focus on the selectivity of counterparties between two banks. Table 1 Panel B shows that if we incorporate network setting in the calculation of hedge cost, the reference firm would switch all its derivative transaction from bank 1 to bank 2 for all three stocks.

This allocation can by justified by comparing the prices of put options on three stocks offered by two banks in Table 2. Panel A suggests that without network spread adjustment, two banks offer the same theoretical put option prices. However, if network impact is considered in the spread adjustments (Panel B), bank 2 provides lower prices of three put options than those offered by bank 1, so that bank 2 has a comparative advantage on hedge cost.

Table 2: Prices of put option for two banks in different network settings Panel A. Price of put options without network spread adjustments

\begin{tabular}{lrr}
\hline Price of put option & Bank 1 & Bank 2 \\
\hline Put option 1 & 0.490908352 & 0.490908352 \\
\hline Put option 2 & 0.067605427 & 0.067605427 \\
\hline Put option 3 & 5.367528166 & 5.367528166 \\
\hline & & \\
\hline & & \\
\hline Panel B. Price of put options with network spread adjustments & Bank 1 \\
\hline Price of put option & 0.510689975 & 0.495817436 \\
\hline Put option 1 & 0.070329652 & 0.068281481 \\
\hline Put option 2 & 5.583817866 & 5.421203447 \\
\hline Put option 3
\end{tabular}

The hedge fraction allocation corresponds to our expectation during parameter setting. Same level of credit rating of both banks indicate equal amount of spread reduction. The reference firm lowers hedge cost by utilizing hedge from a bank with equal or smaller size, bank 2 , since size advantage brings bargaining power and lowers the risk premium. However, the quality of small firms' protection might be under suspicion, since small firms are usually susceptible to outside financial shocks and might be incapable of honoring their contracts. We will investigate these post-contract establishment issues in an a posteriori analysis.

Transparent firm operation of bank 2, compared with the opaque bank 1, makes it more attractive to be a risk taker due to lower informational asymmetry. When link properties are accounted for, the hedge cost advantage of bank 2 becomes more significant. Previous transaction history may provide important information about asset and credit, to each other and enhance trust between counterparties, therefore lowering the premiums. Compared with 
bank 1, bank 2 has a pre-existing business relationship with the reference firm, so that its spread adjustment is lower than that of its competitor.

We conduct some robustness tests to see whether the characteristics of stock holding would affect the hedging strategies in a network setting. We change the correlation between stock returns and find no impact on previous result. It indicates that the features of the network have a much more deterministic impact on hedging strategies, than the relations of stocks in consideration. We also change the initial setting of hedge fraction to see whether the initial value would impact our result. This does not change the optimal solution either.

Overall, our simulation results confirm our initial argument that network features greatly impact hedging strategies through hedge costs. To pursue an optimal hedge ratio, risk managers tend select a hedging partner with cost advantage so that it would preserve firm value. However, the results are based on the default-free assumption, which is unrealistic in practice. In real world, counter-party risk exists and the possibility of contract default should be taken seriously by risk managers. In the following section, we conduct an a posteriori analysis on how network characteristics influence hedging efficacy in the postcontract establishment period.

\section{An A Posteriori Analysis}

In this section, we investigate the efficacy of the hedging contracts in the post-contract establishment period. The main concern of our a posteriori analysis is the counter-party risk, which might result in the inability to fully honor the derivative contract and partially nullify the hedge. In the a priori analysis, we do not consider the default possibility of derivative contracts, which might bring significant loss to the reference firm. For example, the high default possibility of counterparties would result in significant damage to the investment fund, if the fund risk manager only cares about contract costs rather than contract efficacy. Therefore, an optimal hedging strategy cannot be confidently arrived by solely an a priori analysis of risk-return tradeoff. As important as the a priori analysis, an a posteriori study should be conducted to reach a robust hedging decision. We will describe the model structure and a posteriori network setting in the first part, and thereafter conduct result analysis. 


\subsection{Model Structure in the A Posteriori Analysis}

In the a posteriori analysis, we incorporate the counter-party risk in our model and add two additional network features, connectivity $\left(f_{1}\right)$ and ease of link monitoring and enforcement $\left(f_{6}\right)$. These factors are not included in the a priori analysis, since their impact is episodic and is not accounted for in the premium evaluation. We describe how these default-related network features would affect a hedge contract's efficacy and the reference firm's strategies in the post contract establishment period.

Connectivity between firms provides channels for financial contagion, which makes financial institutions susceptible to exogenous shocks. Financial contracts, such as overlapping claims between banks, build the connection between two parties. When one party suffers from financial distress and defaults on its obligations, the other will also suffer losses, which might lead to liquidity problem or financial distress. The complexity of the financial network (i.e. the degree of connectedness between firms) greatly impacts the fragility of financial systems [15]. With an incomplete interbank market and a high degree of interconnectedness, a liquidity shock can be spread by contagion, while a low degree of interconnectedness can shield a firm from contagion. Besides, interbank linkages can also act as "shock-absorbers" so that shocks can be shared and absorbed by other banks. According to Nier et al. [22], after a certain threshold level, connectivity improves the ability of absorption and decreases the probability of contagion. In this paper, we only consider the situation before threshold, which indicates that the firms with higher levels of connectivity are more likely to suffer from external shocks. In the post-contract establishment period, the loss of the counterparty might transfer shocks to the reference firm by default of hedge contract.

The probability of counter-party risk also depends on the ability of risk-taking counterparties to absorb external shocks. Given a certain level of connectivity, a firm with a higher credit rating is more capable of handling the loss from overlapping contracts, absorbing the shocks and preventing the contagion from spreading to the reference firm. On the other hand, low credit firms, which are exposed to many links, are more vulnerable to shocks and have a higher probability to spread financial contagion through its contract links. Therefore, we believe that the connectivity $\left(f_{1}\right)$ and riskiness $\left(f_{2}\right)$ of counterparties interrelate in determin- 
ing the default risk of underlying hedge contract. To describe the impact of connectivity on default probability, we use a multiplier as an indicator of connectivity (as Appendix A). We set the product of connectivity multiplier and theoretical default probability, as dictated by credit rating, as the real default rate of hedge contracts. According to the initial setting for the credit rating of two counterparty banks in the example, theoretical default probabilities of both banks are $0.1 \%$.

Legal infrastructure plays a vital role in corporate default events by providing timely, efficient and impartial resolution. Financial monitoring and enforcement affect the incentives of creditors to monitor, their ability to recontract, as well as creditors' legal rights in reorganization and liquidation. Creditors' rights are important in contracting since law determines who controls the bankruptcy process, who has rights to the property of bankrupt firms and with what priority. Thus recovery rate is impacted by creditors' rights, in other words, the legal enforcement and monitoring. To describe this influence, we add another link factor, ease of link monitoring and enforcement $\left(f_{6}\right)$, as a percentage of credit-rating-decided recovery rate, to evaluate the total recovery rate of firms. As described in Appendix A, the recovery rate percentage of banks is an increasing function of level of link monitoring and enforcement. In the specific example, the same credit ratings of two banks provide the same theoretical recovery rate, $59.29 \%$, but in practice the true value of recovery rates would be different due to different network features of counterparties.

In the a posteriori analysis, we focus on the impact of counter-party risk on the hedge contracts. Since the default risk greatly threatens hedge contract's efficacy, whether the reference firm will maintain the optimal strategy as dictated by the a priori analysis, or consider a modification to the hedging strategy depends on counterparties' a posteriori network features. Given the a priori optimal strategy, fully hedged by the bank 2, we study how the hedging risk and reference firm value would change with different settings of the a posteriori features, connectivity $\left(f_{1}\right)$ and ease of link monitoring and enforcement $\left(f_{6}\right)$ of its counterparties. To measure the efficacy of the hedge contract in the post-contract establishment period, the firm payoff equation $\left(\tilde{Y}_{\text {hedge }_{p}}\right)$ is modified from the a priori one by adding 
indicators of default probability and recovery rate as below,

$$
\begin{array}{r}
\tilde{Y}_{\text {hedge }_{p}=}=\sum_{i=1}^{3} \sum_{j=1}^{2} P_{r j} z_{i} x_{i j}\left(S_{i}(0) \tilde{R}_{i}+\left(K_{i}-S_{i}(0) \tilde{R}_{i}\right)_{+}\right)+\sum_{i=1}^{3} \sum_{j=1}^{2}\left(1-P_{r j}\right) z_{i} x_{i j} S_{i}(0) \tilde{R}_{i}+ \\
\sum_{i=1}^{3} z_{i}\left(1-\sum_{j=1}^{2} x_{i j}\right) S_{i}(0) \tilde{R}_{i} .
\end{array}
$$

where $P_{r j}$ is a percentage denoting recovery rate of the bank $j$, quantified by the theoretical recovery rate, decided by credit rating $\left(f_{2}\right)$, times its recovery rate percentage, measuring the ease of link monitoring and enforcement $\left(f_{6}\right)$. When a default occurs on the bank $j, P_{r j}$ of risk exposure hedged by contract would be honored, leaving the rest $\left(1-P_{r j}\right)$ exposed to equity risk. Thus the firm's payoff in the a posteriori analysis is formed by three parts: honored contract value, the unhonored one and unhedged equity risk. In the simulation, we use the Bernoulli Distribution to indicate the occurrence of default event, where the overall default probability is measured by the product of connectivity multiplier $\left(f_{1}\right)$ and default probability given by credit rating $\left(f_{2}\right)$. If there is no default, recovery rate is set at $100 \%$.

The expected firm value is calculated as eqn(4), the discounted firm payoff minus hedge contract cost, $\tilde{V}_{\text {hedge }_{p}}=\frac{\tilde{Y}_{h_{e d g e p}}}{1+d}-H$. To measure the efficacy of the hedge contract, we follow the objective function used in the a priori analysis,

$$
\sigma_{\text {hedge }}^{2}+\left(E\left[\tilde{V}_{\text {no-hedge }}\right]-E\left[\tilde{V}_{\text {hedge }_{p}}\right]\right)
$$

In the a priori analysis, bank 2 is chosen as the only risk taker because it provides cheaper risk coverage in a no-default scenario. If counter-party risk is considered, we inquire whether the previous optimal strategy is still the best one. To answer this question, we compare various values in perfect hedge and imperfect hedge situations and show how worse off the contract might finally become due to default. Here by perfect hedge we mean the ideal world without default, while the existence of counter-party risk makes for an imperfect hedge. 


\subsection{Result of the A Posteriori Analysis}

Since bank 2 is the only one taking the reference firm's risk through the a priori analysis, we study how its a posteriori network features would impact the hedge contract efficacy. According to the parameter setting in the previous part, the reference firm's value is supposed to be higher if a counterparty has a lower degree of connectivity (lower default probability multiplier) and a higher level of link monitoring and enforcement (higher recovery percentage). We will focus on two extreme cases: bank 2 with the worst network features (high default probability multiplier and low recovery percentage) and the best ones (low default probability multiplier and high recovery percentage). Through analysis of the extreme cases, we can get a brief idea of how the a posteriori network features can affect hedge efficacy in an imperfect world. Especially the worst case will tell us what a seemingly perfect hedge strategy would bring to the reference firm if counter-party risk is ignored. Based on the Monte Carlo simulation of return distributions $(R)$ with a sample size of $5 \times 10^{6}$, we calculate the value of the a posteriori objective function. We also compute the variance of hedged

firm present value, the difference of expected firm value in unhedged and hedged situations in two extreme cases.

Table 3, Panel A shows the results when bank 2 has few links with other firms (quantified by default probability multiplier 1) and under strict monitoring of legal infrastructure (quantified by recovery percentage 100\%). Panel B shows the worst case, that bank 2 is highly connected (quantified by default probability multiplier 2) but under loose monitoring (quantified by recovery percentage $70 \%$ ). Corresponding to our expectation, in both cases the values of objective function is higher than that of the perfect hedge situation. This means the hedging contract become less effective if counter-party risk exists. This negative impact is more significant if bank 2 has the highest probability to breach the contract and the lowest level of legal infrastructure monitoring as Panel B. Objective function value increases $0.0125 \%$ from the perfect hedge to the imperfect hedge, mainly from higher variance of firm value since it dominates the objective function.

Comparing the percentage change of variance in two panels, we find the change in Panel B $(0.0125 \%)$ is around 16.33 times that of Panel A $(0.0008 \%)$, which suggests that a posteriori 
Table 3: The reference firm's hedge contract efficacy in perfect hedge and imperfect hedge

\begin{tabular}{|c|c|c|c|c|}
\hline & \multicolumn{4}{|c|}{ Fully Hedged by 2nd bank } \\
\hline & perfect hedge & imperfect hedge & $\begin{array}{l}\text { absolute value } \\
\text { difference between } \\
\text { imperfect one and } \\
\text { perfect one }\end{array}$ & percentage difference \\
\hline Objective function value & $3,712,020,804.64328$ & $3,712,049,284.28689$ & $28,479.64361$ & $0.0008 \%$ \\
\hline $\begin{array}{l}\text { Variance of present firm } \\
\text { value }\end{array}$ & $3,712,014,368.76315$ & $3,712,042,848.13302$ & $28,479.36987$ & $0.0008 \%$ \\
\hline $\begin{array}{l}\text { Expected firm value } \\
\text { difference between } \\
\text { unhedged and hedged } \\
\text { situatation }\end{array}$ & $6,435.88013$ & $6,436.15387$ & 0.27374 & $0.0043 \%$ \\
\hline VaR & $295,013.01727$ & $295,011.93770$ & -1.07957 & $-0.0004 \%$ \\
\hline CVaR & $289,878.12913$ & $289,877.14038$ & -0.98875 & $-0.0003 \%$ \\
\hline
\end{tabular}

Panel B. 2nd bank has highest level of connectedness and lowest degree of link monitoring and enforcement

\begin{tabular}{|c|c|c|c|c|}
\hline & \multicolumn{4}{|c|}{ Fully hedged by 2 nd bank } \\
\hline & perfect hedge & imperfect hedge & $\begin{array}{l}\text { absolute value } \\
\text { difference between } \\
\text { imperfect one and } \\
\text { perfect one }\end{array}$ & percentage difference \\
\hline Objective function value & $3,712,020,804.64328$ & $3,712,485,835.89996$ & $465,031.25668$ & $0.0125 \%$ \\
\hline $\begin{array}{l}\text { Variance of present firm } \\
\text { value }\end{array}$ & $3,712,014,368.76315$ & $3,712,479,395.99195$ & $465,027.22880$ & $0.0125 \%$ \\
\hline $\begin{array}{l}\text { Expected firm value } \\
\text { difference between } \\
\text { unhedged and hedged } \\
\text { situatation }\end{array}$ & $6,435.88013$ & $6,439.90801$ & 4.02787 & $0.0626 \%$ \\
\hline VaR & $295,013.01727$ & $294,998.91365$ & -14.10362 & $-0.0048 \%$ \\
\hline CVaR & $289,878.12913$ & $289,851.85355$ & -26.27558 & $-0.0091 \%$ \\
\hline
\end{tabular}


features of the counterparty do influence hedging contract efficacy to a certain degree. We also take a look at another component of objective function besides variance, the percentage change of difference of expected firm value in unhedged and hedged situations. A 0.0043\% drop in Panel A and a $0.0626 \%$ drop in Panel B suggest that a posteriori features have a greater impact on expected firm value than on variance.

To further investigate how the a posteriori network features affect the hedging contract, we turn to other risk measures besides variance. The histogram of the simulated hedged firm value shows fat right tail skew instead of a symmetric normal distribution (Figure 2). Since variance is a central dispersion measure of risk, which may not be a complete indicator of risk for an asymmetric distribution, we pick two other measures to obtain a better understanding of how risk changes in an imperfect environment. Both absolute Value-at-Risk (VaR) and Conditional Value-at-Risk $(C V a R)$ are measures of tail risk, indicating how much loss a firm may suffer in a bad situation.

Figure 2: Histogram of simulated hedged firm value

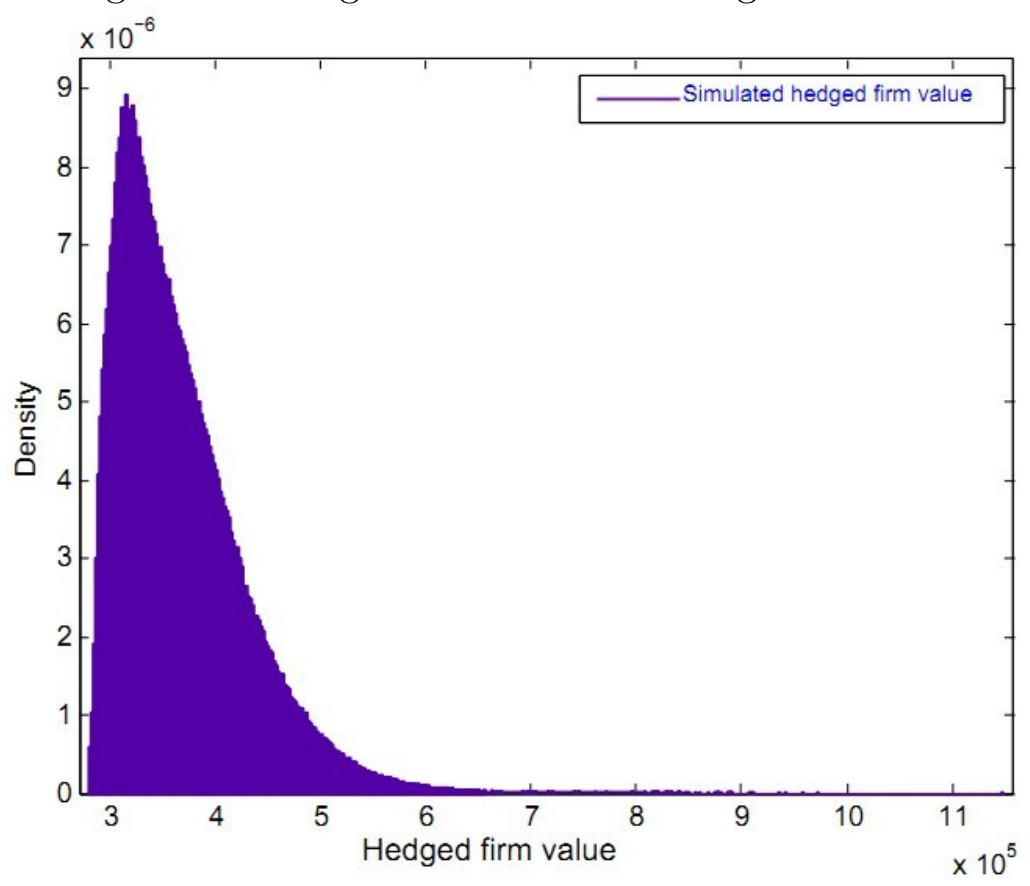

Value-at-Risk $(\mathrm{VaR})$ is a widely used risk measure of tail risk on a specific portfolio of financial assets. Following the general definition of absolute $V a R$, we calculate the threshold 
of firm value at 5 percentile, which tells us with $5 \%$ probability the firm value would drop below the threshold. Conditional Value-at-Risk $(C V a R)$ is the expected firm value lower than $V a R$, calculated by $E\left[\tilde{V}_{\text {Hedge }} \mid \tilde{V}_{\text {Hedge }}<\operatorname{VaR} 5 \%\right]$. Similar to the results based on the variance measure, both $V a R$ and $C V a R$ display a small amount of decrease from a perfect situation to an imperfect one. However, when comparing the percentage change in two cases, we find that of absolute $\operatorname{VaR}$ in Panel B $(-0.0048 \%)$ is around 13.06 times of that in Panel A $(-0.0004 \%)$, and 26.57 times in the case of $C V a R$.

These results shed some light how influential the a posteriori features, connectivity and the ease of link monitoring and enforcement, are on the reference firm's hedging strategy. From the above analysis, we conclude that bank 2 is not a perfect risk taker in an imperfect hedge situation, especially when it has the highest degree of connectivity and the weakest legal monitoring and enforcement.

Now we might wonder the following questions, what would happen if bank 1 is excellent in dealing with default risk, whether transferring certain amount of risk to bank 1 would improve the hedge efficacy, what risk percentage is the most feasible to transfer to bank 1 . To answer those questions, we analyze all possible cases, in which both risk takers have three levels of connectedness and three levels of link ease of monitoring and enforcement. The number of combinations of a posteriori network characteristic for two banks is 81 total (9 posteriori network feature combinations of bank $1 \times 9$ posteriori network feature combinations of bank 2). Besides, to compare the hedging contract efficacy in various contract settings, we conduct the following five risk allocation strategies:

1. $100 \%$ hedged by bank 2

2. $20 \%$ hedged by bank 1 and $80 \%$ hedged by bank 2

3. $50 \%$ hedged by bank 1 and $50 \%$ hedged by bank 2

4. $80 \%$ hedged by bank 1 and $20 \%$ hedged by bank 2

\section{5. $100 \%$ hedged by bank 1}

for each a posteriori network feature combination. The design of risk allocation is from the idea that what would happen if more and more risk is taken by bank 1 . We apply various 
measures on the contract efficacy, including the value of objective function, variance of firm value, absolute $V a R$ and $C V a R$ of hedged firm value. To clarify our method and explain the results, we discuss 9 out of 81 cases in detail as examples, and then summarize the overall results for all 81 cases.

In the 9 cases, bank 1 is set as an ideal hedge partner in dealing with default risk, which has a low level of connectedness (quantified by default probability multiplier 1) and a high level of link monitoring and enforcement (quantified by recovery percentage 100\%); bank 2 is set at 9 different combinations of posteriori features ( 3 levels of connectedness $\times 3$ levels of link monitoring and enforcement). We expect that in these situations, it is most likely that the reference firm would benefit from switching certain amount of risks to bank 1 .

Figure 3: Values of Objective function in different risk allocation strategies and under different network features

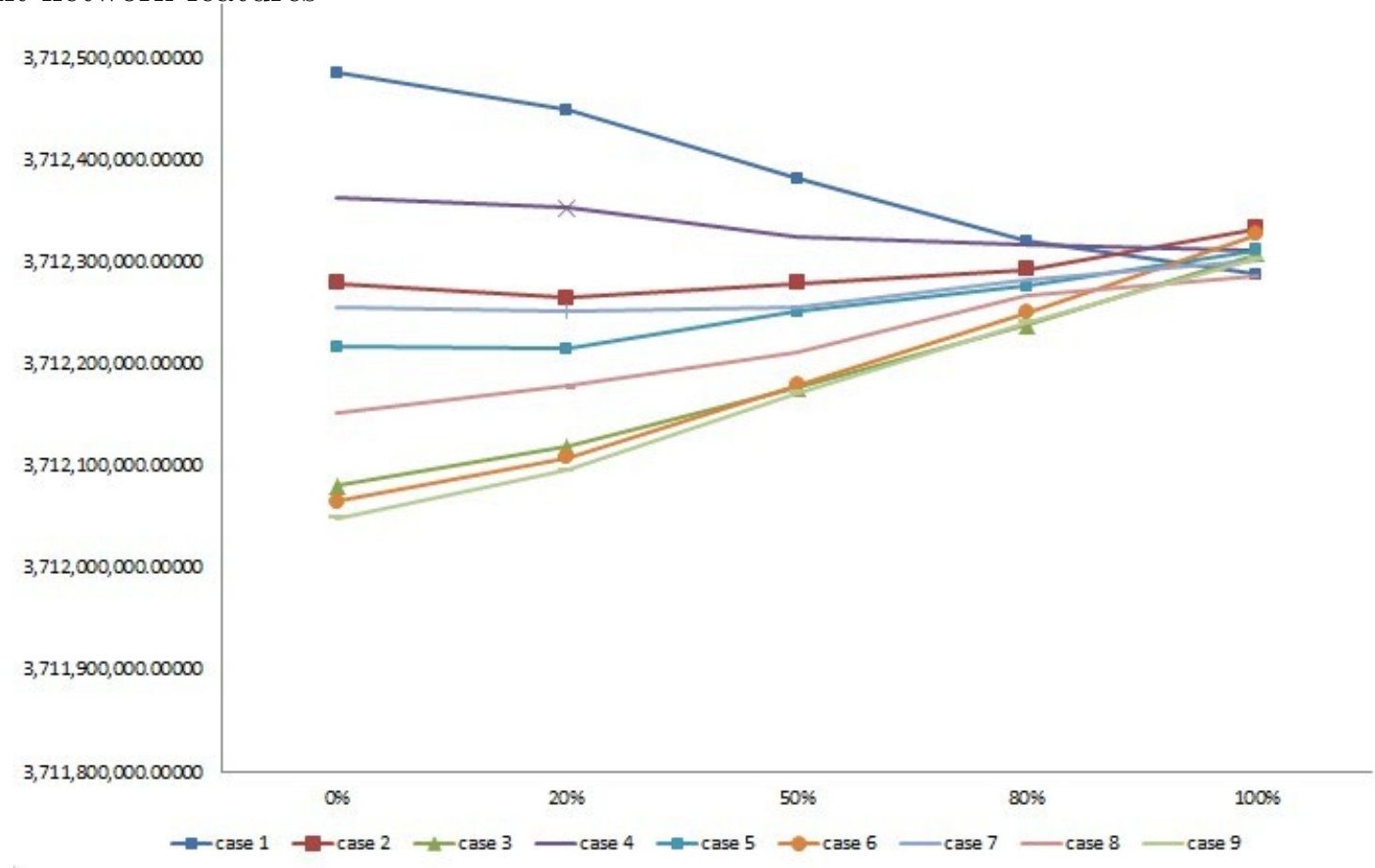

Figure 3 shows us how the value of objective function changes with different risk allocation in the 9 cases. There are two curves (case 1 and 4) display a downward trend, indicating lower risk of reference firm; three curves (case 2, 5 and 7) display U-shape, which suggest the risk level reverse after reaching certain level; and the rest four (case 3, 6, 8 and 9) maintain 
an upward trend, meaning higher risk. All the curves approach to the similar level at $100 \%$.

Here we give a detailed description of Figure 3. When bank 2 has the lowest level of legal monitoring and enforcement, no matter what level of connectedness it has, the reference firm benefits if $20 \%$ risk is taken by bank 1 (case 1, 4 and 7). It also happens when bank 2 is in a medium level of link monitoring, and medium/high levels of connectedness (case 2 and 5 ). However, when a higher percentage of risk $(50 \%)$ is covered by bank 1 , only in two out of five cases (case 1 and 4) the objective function value drops to a lower level, while the others reverse to even higher values than that in the original optimal strategy (fully-hedged-by-bank 2). Further, when the percentage of risk taken by bank 1 is increased to $80 \%$ and even $100 \%$, the objective function value keeps declining for case 1 and 4, and for case 2, 5 and 7 it raises to higher levels. For the rest four cases (case 3, 6, 8 and 9), we find the contract efficacy is exacerbated by greater percentage of risk taken by bank 1 . We give the calculation details in Appendix B, Table 4-I.

We turn to other measures of risks, absolute $V a R$ and $C V a R$ at 5 percentile. According to the report of Table 4-III and Table 4-IV in Appendix B, there is no evidence showing that the reference firm would benefit from more risk taken by bank 1 since both absolute $\operatorname{VaR}$ and $C V a R$ drop off gradually, which means the reference firm suffers greater tail risk.

In the above analysis of the 9 cases, we find some evidence that in an imperfect world, the a posteriori network features do impact corporate hedging contract efficacy through contract default rate and recovery rate. Specifically, when bank 1 has the lowest degree of connectedness and the highest level of link monitoring, bank 2 has the lowest level of link monitoring, and medium/high levels of connectivity, the risk exposure curve displays a declining trend, which seems to be contrary to the a priori optimal hedging decision fully hedged by bank 2 . In the other three cases, low in linkage numbers and a low degree of monitoring, a medium level of monitoring and medium/high levels of connectedness, the risk exposure curve displays a U-Shape, suggesting a small amount (20\%) of risk switching is beneficial but excessive amount is detrimental. However, according to other measures of risk, absolute $V a R$ and $C V a R$, the reference firm suffers greater tail risk if increasing amount of risk is taken by bank 1. It suggests that managers, who are focusing on alleviating low tail risks, are unlikely to consider modification of their hedging strategy in an imperfect world. 
Following the same method, we analyze all 81 cases with different combinations of a posteriori features of the two banks. Figure 4 shows the fraction of trends displayed by risk objectives. There are 11 out of 81 (13.58\%) cases where 20\%-risk-switch strategy benefits the reference firm by lowering risk. In the 11 cases, 6 of them (7.41\%) would experience reverse risk exposure when more risks, $50 \%$ and higher percentage, are taken by bank 1, and in the other 5 cases $(6.17 \%)$ risk keeps declining. Thus with $86.42 \%$ probability the contract efficacy would take a turn for the worse if deviating from the original optimal strategy.

Figure 4: The probability distribution of risk objective curve trend

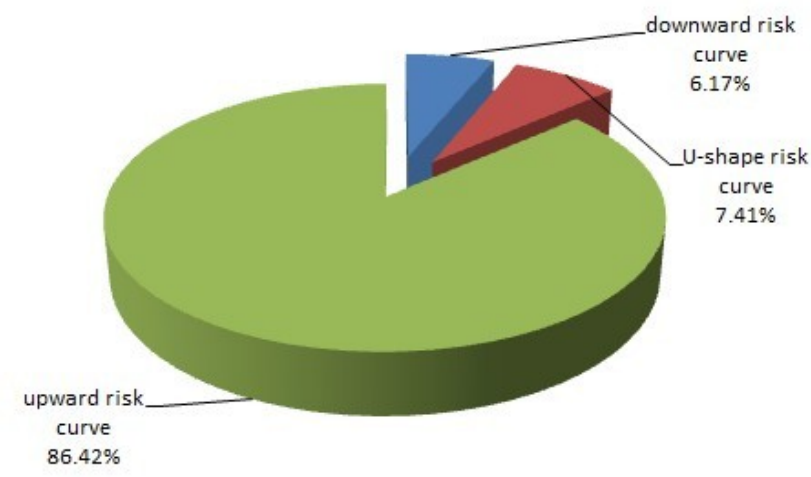

In the five cases with downward trend objective function, two of them are already been discussed in the previous paragraphs. The other three occur when bank 2 has undesirable a posteriori network features, high numbers of linkages and a low level of link monitoring; at the same time bank 1 has low connectedness and medium/low monitory multipliers, or medium connectedness and high link monitoring. In the six cases with U-shape objective function, four of them are under the situation when bank 2 has the least desirable a posteriori network features. Thus we conclude that when a counterparty has a high chance to default and fail to fully honor the contract, it is not wise to put all the eggs into one basket.

On the other hand, we do not find any case showing switch risks would improve tail risks by absolute $\operatorname{VaR}$ and $C V a R$. Therefore, the risk management decision depends on what kind of risk diminishing goal the firm has. If the volatility of firm value is the priority, there is a certain degree of chance that original hedging decision, fully-hedged-by-bank 2, would become suboptimal, and small amount of risk covered by bank 1 is desirable. If reducing tail 
risk is the object, the firm should still keep their original strategy since any switch would make it worse off.

\section{Discussion and Conclusions}

In this paper, we derive and assess a model towards creation of a framework in which a firm's financial environment is an integral part of arriving at the optimal hedging decisions. In the framework, the characteristics of firms in a network and their interconnectedness affects the cost of hedging contracts, as well as the efficacy of protection in the post-contract establishment period.

We develop a specific model for an investment fund's decision making in transferring three types of equity risk to a set of banks and obtain its optimal hedging decision based on a risk-return tradeoff analysis. We find the cost of protection greatly influences the willingness of a firm entering a hedge contract, that cost advantage might lead a potential risk taker to take a dominant role in the degree of protection offered. Our framework is also useful for modeling and measuring counter-party risk, which are studied in the a posteriori analysis by including two extra network factors as the environmental impact on default probability and recovery rate. We find the "optimality" of a hedge contract in the a posteriori analysis depends on the objective and the risk measures. This means firms focusing on minimizing variance of firm value might consider a small deviation from a priori optimal strategy, those paying more attention on tail risk tend to stay with the a priori optimal strategy.

In the a priori analysis, we conduct a risk-return analysis and capture network features in the evaluation of hedge costs. Under the specific setting of financial environment faced by the investment fund, the optimal hedging strategy is fully-hedged-by-bank 2 , since it has a price advantage compared to another bank. It suggests that the financial environment influences corporate hedging decisions through contract costs and risk takers with a cost advantage take a dominant role in the degree of protection offered.

However, only considering risk-return tradeoff in the a priori analysis is insufficient to measure hedging contract efficacy, hence we further investigate the issue in post-contract establishment periods. Two extra network features, connectivity and the ease of link mon- 
itoring and enforcement, are added to the model to describe how a network environment would affect a firm's hedging decision and contract efficacy through impact on counter-party risk. By analyzing various a posteriori network feature combinations (81 totally) and the contract efficacy in five different risk distribution strategies among two banks, we find that in 11 out of 81 cases (with $13.58 \%$ probability) if the investment fund modifies its a priori strategy (fully-hedged-by-bank 2) to a 20\%-risk-hedged-by-bank 1 strategy, the contract efficacy can be improved. The benefits are most likely to happen when bank 2 has the highest degree of connectivity and the weakest legal monitoring and enforcement. However, excessive risk switch, $50 \%$ and higher, would turn the contract efficacy to the worse with $7.41 \%$ probability. Overall there is $86.42 \%$ chance that any deviation from the a priori optimal strategy would lower the contract efficacy. These results are obtained based on a corporate risk measure - variance of firm value.

When we conduct our analysis based on tail risk measures, absolute $\operatorname{VaR}$ and $C V a R$, the result does not suggest any benefit from deviation from the a priori optimal decision. $\operatorname{VaR}$ and $C V a R$ show that firm's tail risk increases if more risk is covered by bank 1 , which suggests that a firm aiming at reducing tail risks is unlikely to deviate from fully-hedged-bybank 2 strategy.

Our analysis points to optimal hedging decision under a network framework. Our approach, which directly relates various network characteristics with contract costs and postcontract protection efficacy, provides the initial insights of how the financial environment affects firms' risk management strategies, and a basis for complicated models in future studies. By making it explicit how network factors affect spreads in the a priori analysis, and the default probability and recovery rate in the a posteriori analysis, our approach is the first to offer specific guidance to risk managers on how the corporate financial environment should be considered in hedging decision-making. In developing our story for corporate risk management under networked environment, we can extend the analysis to various types of risks (currency risk, interest risk, etc.) and a number of hedging instruments (derivative combinations, insurance, etc.) used in real world. We can also study more sophisticated corporate network maps. These are promising directions of future research. 


\section{Appendix A: Hedge Factors Description}

\subsection{Node Properties}

$f_{1}$ : Connectivity of Firm. Connectivity is quantified as the multiplier of default probability. This factor is used in our a posteriori analysis and can't be priced in the a priori analysis. It is supposed that the high connectedness of a firm would bring channels so that the probability of suffering from exogenous shocks is increased. We interrelate it with credit rating $\left(f_{2}\right)$ since low credit rated firms are more vulnerable to external shocks than high rated ones. Therefore, the probability of default would rise if a highly risky firm is exposed to too many links. We consider three levels of firm connectivity, Low Connectivity $\{0\}$, Medium Connectivity $\{1\}$ and High Connectivity $\{2\}$. We assume no multiplier effect while few external links, but double the effect if a firm is highly connected.

$\{\mathbf{0}\} \rightarrow$ Multiplier of default probability $=1$.

$\{1\} \rightarrow$ Multiplier of default probability $=1.5$.

$\{2\} \rightarrow$ Multiplier of default probability $=2$.

$f_{2}$ : Riskiness. This captures counter-party risk and recovery rate of each firm. We create three groups by credit ratings. The spread is modeled as a decreasing function of credit rating. The default probability and recovery rate for each credit rating level is approximated from those of corporate bond issuers in the statistical review of Moody's, 1920-2003.

$\mathbf{A A A} / \mathbf{A A} \rightarrow 0 \%$; Probability of default $=0$; Recovery rate $=34.8 \%$.

$\mathrm{A} / \mathbf{B B B} \rightarrow-1 \%$; Probability of default $=0.1 \%$; Recovery rate $=59.29 \%$.

BB and Below $\rightarrow-2 \%$; Probability of default $=5 \%$; Recovery rate $=87.32 \%$.

$f_{3}$ : Size of Firm. We consider four levels of firm size, Small $\{0\}$, Medium $\{1\}$, Large $\{2\}$ and Very Large $\{3\}$. Instead of firm size in isolation, we consider the difference in firm size between the risk taker firm and the risk transferring firm as an indicator of spread. The spread is modeled as an increasing function of differential of firm size. 
$\{-3,-2,-1,0\} \rightarrow 0 \%$

$\{1\} \rightarrow 1 \%$

$\{2\} \rightarrow 2 \%$

$\{3\} \rightarrow 3 \%$

$f_{4}$ : Transparency of Firm Operations. Lack of transparency of either firm increases informational asymmetry, as well as spreads. We consider four levels of transparency, Very Opaque $\{0\}$, Opaque $\{1\}$, Transparent $\{2\}$ and Very Transparent $\{3\}$. Since both firm's transparency affects the contractual terms, the sum of transparency of two node firms in the same link is mapped to the spreads as a decreasing function.

$\{6,5,4\} \rightarrow 0 \%$

$\{3,2\} \rightarrow 1 \%$

$\{1,0\} \rightarrow 2 \%$

\subsection{Link Properties}

$f_{5}$ : Age of Link. Longer transactional relationship implies the possibility of volume discounts, greater amount of information of the reference firm's assets and credit history. We consider four levels, First Time, Short Period, Medium, and Long-term transactional relationship between the firms. The spread is modeled as a decreasing function of the the age of link.

First Time $\rightarrow 1 \%$

Short Period $\rightarrow 0 \%$

Medium/Long Period $\rightarrow-1 \%$

$f_{6}$ : Ease of Link Monitoring and Enforcement. Legal infrastructure can support recovery, in case of breach in contractual terms. We consider three levels of link monitoring and enforcement, Low $\{0\}$, Medium $\{1\}$ and High $\{2\}$. The variable is quantified as a 
percentage of theoretical recovery rate and the maximum value is $100 \%$. This factor will be used for the a posteriori analysis to determine the scope of recovery rate in the event of default.

Low $\rightarrow$ Percentage of recovery rate $=70 \%$.

Medium $\rightarrow$ Percentage of recovery rate $=85 \%$.

High $\rightarrow$ Percentage of recovery rate $=100 \%$. 


\section{Appendix B: Tables}

\section{Table 3-I. Objective function values in five risk allocation strategies}

The a posteriori network features of the bank 1 is set as the lowest level of connectivity and the highest level of ease of link monitoring and enforcement. The table displays the objective function values under various settings of the bank 2 a posteriori network features. The columns represent three levels of connectivity of the bank 2 and rows represent three levels of ease of link monitoring and enforcement of the bank 2. Panel A - Panel D report results of five risk allocation strategies. For certain network feature setting of two banks, we compare the values of objective function in five panels and highlight those lower than the corresponding values in Panel A (the optimal strategy in a priori analysis). Those highlighted numbers indicate deviation from fully-hedge strategy is beneficial, which are discussed in the article. 
Panel A. 100\% risk covered by 2nd bank

\begin{tabular}{|c|c|c|c|c|}
\hline & & \multicolumn{3}{|c|}{ Connectivity } \\
\hline & & low & medium & high \\
\hline \multirow{3}{*}{$\begin{array}{c}\text { Ease of link monitoring } \\
\text { and enforcement }\end{array}$} & low & $3,712,256,416.29800$ & $3,712,362,904.14149$ & $3,712,485,835.89996$ \\
\hline & medium & $3,712,151,697.72288$ & $3,712,217,687.14614$ & $3,712,279,182.84468$ \\
\hline & high & $3,712,049,284.28689$ & $3,712,065,646.47388$ & $3,712,080,783.00397$ \\
\hline \multicolumn{5}{|c|}{ PanelB. $20 \%$ risk covered by 1 st bank, $80 \%$ risk covered by 2 nd bank } \\
\hline & & \multicolumn{3}{|c|}{ Connectivity } \\
\hline & & low & medium & high \\
\hline \multirow{3}{*}{$\begin{array}{c}\text { Ease of link monitoring } \\
\text { and enforcement }\end{array}$} & low & $3,712,252,087.43586$ & $3,712,353,104.08412$ & $3,712,449,193.99635$ \\
\hline & medium & $3,712,178,655.80372$ & $3,712,214,822.48253$ & $3,712,264,886.20259$ \\
\hline & high & $3,712,095,905.81854$ & $3,712,109,144.56383$ & $3,712,118,998.53523$ \\
\hline
\end{tabular}

PanelC. 50\% risk covered by 1 st bank, 50\% risk covered by 2 nd bank

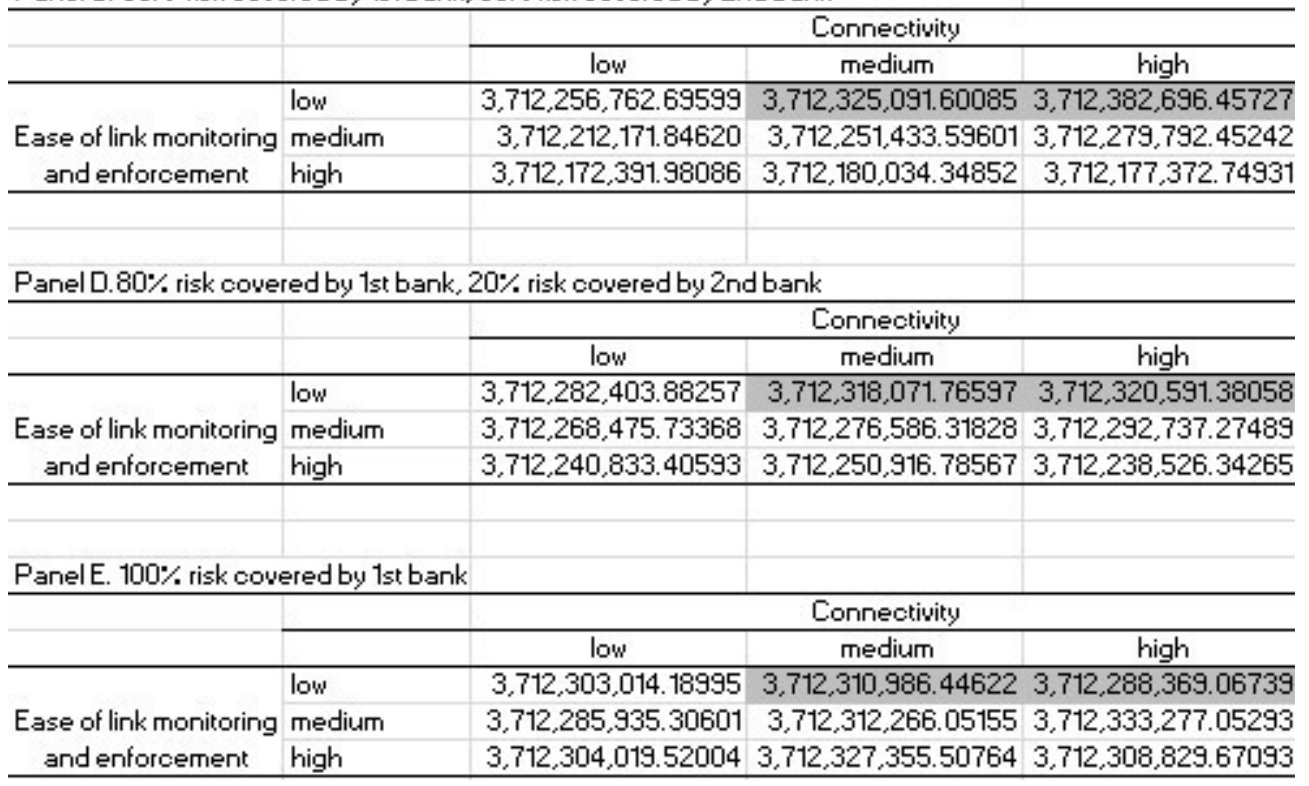




\section{Table 3-II. Variances of firm present value in five risk allocation strategies}

The a posteriori network features of the bank 1 is set as the lowest level of connectivity and the highest level of ease of link monitoring and enforcement. The table displays variances of firm present value under various settings of the bank 2 a posteriori network features. The columns represent three levels of connectivity of the bank 2 and rows represent three levels of ease of link monitoring and enforcement of the bank 2. Panel A - Panel D report results of five risk allocation strategies. At certain network feature setting of two banks, we compare the values of variance of firm value in five panels.

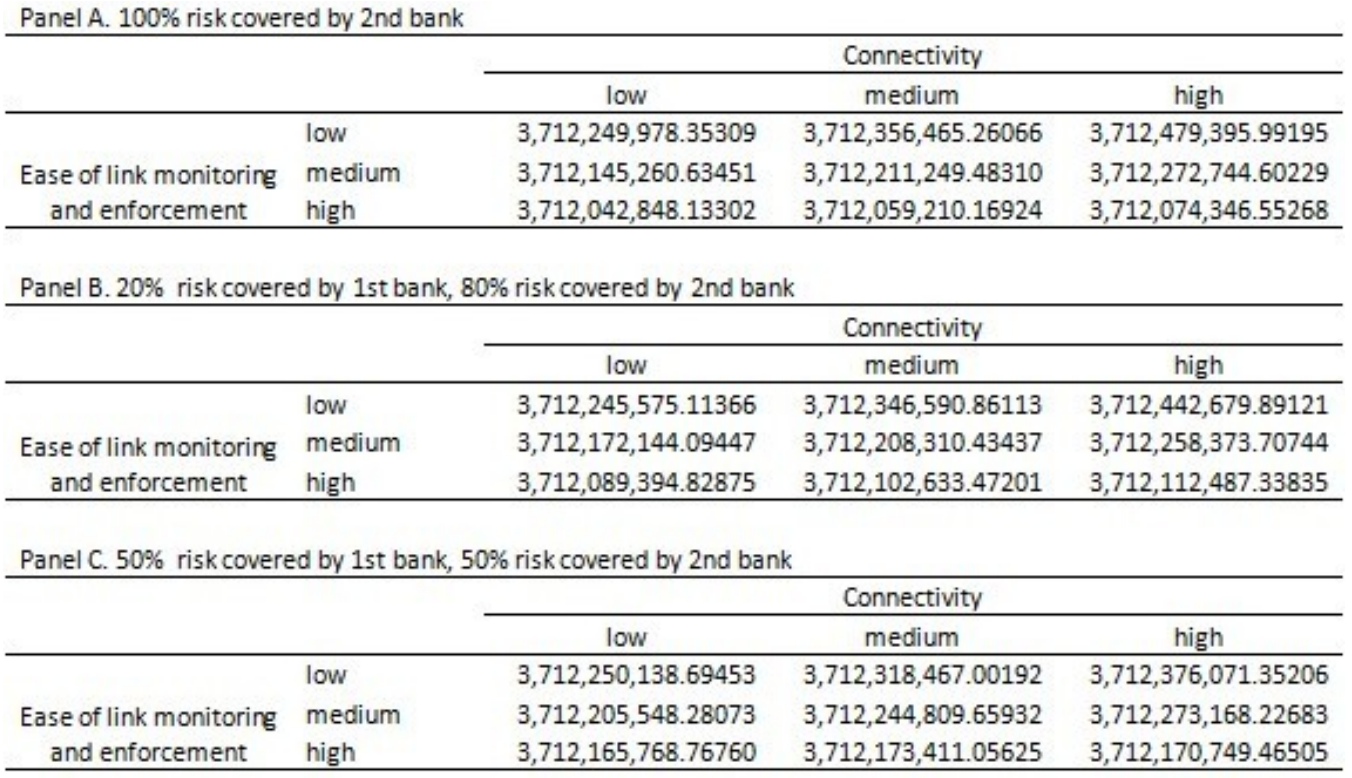

Panel D. $80 \%$ risk covered by 1 st bank, $20 \%$ risk covered by 2 nd bank

\begin{tabular}{clccc}
\hline & & \multicolumn{3}{c}{ Connectivity } \\
\cline { 3 - 5 } & & low & medium & high \\
\hline & low & $3,712,275,668.17940$ & $3,712,311,335.73897$ & $3,712,313,855.29827$ \\
Ease of link monitoring & medium & $3,712,261,740.16209$ & $3,712,269,850.69160$ & $3,712,286,001.45146$ \\
and enforcement & high & $3,712,234,098.05406$ & $3,712,244,181.33723$ & $3,712,231,791.02054$ \\
\hline
\end{tabular}

Panel E. $100 \%$ risk covered by 1st bank

\begin{tabular}{cllcc}
\hline & \multicolumn{4}{c}{ Connectivity } \\
\cline { 2 - 5 } & low & medium & high \\
\hline \multirow{2}{*}{$\begin{array}{c}\text { Ease of link monitoring } \\
\text { and enforcement }\end{array}$} & medium & $3,712,296,203.97098$ & $3,712,304,176.19486$ & $3,712,281,558.98650$ \\
& high & $3,712,279,125.23786$ & $3,712,305,455.76082$ & $3,712,326,466.63845$ \\
\hline
\end{tabular}




\section{Table 3-III. Absolute Value-at-Risk in five risk allocation strategies}

The a posteriori network features of the bank 1 is set as the lowest level of connectivity and the highest level of ease of link monitoring and enforcement. The table displays absolute Valueat-Risk(VaR) under various settings of the bank 2 a posteriori network features. The columns represent three levels of connectivity of the bank 2 and rows represent three levels of ease of link monitoring and enforcement of the bank 2. Panel A - Panel D report results of five risk allocation strategies. At certain network feature setting of two banks, we compare the absolute VaR value in five panels.

Panel A. $100 \%$ risk covered by 2 nd bank

\begin{tabular}{clrrr}
\hline & & & \multicolumn{2}{c}{ Connectivity } \\
\cline { 3 - 5 } & & low & medium & high \\
\hline & low & $295,006.92$ & $295,002.52$ & $294,998.91$ \\
Ease of link monitoring & medium & $295,008.66$ & $295,007.26$ & $295,005.86$ \\
and enforcement & high & $295,011.94$ & $295,011.31$ & $295,010.97$ \\
\hline
\end{tabular}

Panel B. $20 \%$ risk covered by 1 st bank, $80 \%$ risk covered by 2 nd bank

\begin{tabular}{|c|c|c|c|c|}
\hline & & \multicolumn{3}{|c|}{ Connectivity } \\
\hline & & low & medium & high \\
\hline \multirow{3}{*}{$\begin{array}{c}\text { Ease of link monitoring } \\
\text { and enforcement }\end{array}$} & low & $294,931.74$ & $294,928.93$ & $294,924.72$ \\
\hline & medium & $294,934.12$ & $294,932.95$ & $294,931.44$ \\
\hline & high & $294,935.71$ & $294,936.06$ & $294,935.32$ \\
\hline \multicolumn{5}{|c|}{ Panel C. $50 \%$ risk covered by 1 st bank, $50 \%$ risk covered by 2 nd bank } \\
\hline & & \multicolumn{3}{|c|}{ Connectivity } \\
\hline & & low & medium & high \\
\hline \multirow{3}{*}{$\begin{array}{c}\text { Ease of link monitoring } \\
\text { and enforcement }\end{array}$} & low & $294,820.51$ & $294,817.96$ & $294,816.93$ \\
\hline & medium & $294,821.65$ & $294,820.70$ & $294,819.55$ \\
\hline & high & $294,822.53$ & $294,822.11$ & $294,822.08$ \\
\hline
\end{tabular}

Panel D. $80 \%$ risk covered by 1 st bank, $20 \%$ risk covered by 2 nd bank

\begin{tabular}{clrrr}
\hline & & \multicolumn{3}{c}{ Connectivity } \\
\cline { 3 - 5 } & & low & medium & high \\
\hline & low & $294,708.31$ & $294,706.58$ & $294,706.72$ \\
Ease of link monitoring & medium & $294,707.96$ & $294,708.60$ & $294,707.38$ \\
and enforcement & high & $294,709.29$ & $294,709.59$ & $294,709.75$ \\
\hline
\end{tabular}

Panel E. $100 \%$ risk covered by 1 st bank

\begin{tabular}{clrrr}
\hline & \multicolumn{4}{c}{ Connectivity } \\
\cline { 2 - 5 } & & low & medium & high \\
\hline \multirow{2}{*}{ Ease of link monitoring } & medium & $294,633.60$ & $294,633.60$ & $294,633.41$ \\
and enforcement & high & $294,633.40$ & $294,632.20$ & $294,632.21$ \\
\hline
\end{tabular}




\section{Table 3-IV. Conditional Value-at-Risk in five risk allocation strategies}

The a posteriori network features of the bank 1 is set as the lowest level of connectivity and the highest level of ease of link monitoring and enforcement. The table displays Conditional Valueat-Risk(CVaR) under various settings of the bank 2 a posteriori network features. The columns represent three levels of connectivity of the bank 2 and rows represent three levels of ease of link monitoring and enforcement of the bank 2. Panel A - Panel D report results of five risk allocation strategies. At certain network feature setting of two banks, we compare the CVaR value in five panels.

Panel A. $100 \%$ risk covered by 2 nd bank

\begin{tabular}{clcrr}
\hline & & & \multicolumn{2}{c}{ Connectivity } \\
\cline { 3 - 5 } & & low & medium & high \\
\hline & low & $289,865.41$ & $289,859.32$ & $289,851.85$ \\
Ease of link monitoring & medium & $289,871.73$ & $289,868.18$ & $289,865.76$ \\
and enforcement & high & $289,877.14$ & $289,876.29$ & $289,875.52$ \\
\hline
\end{tabular}

Panel B. $20 \%$ risk covered by 1 st bank, $80 \%$ risk covered by 2 nd bank

\begin{tabular}{|c|c|c|c|c|}
\hline & & \multicolumn{3}{|c|}{ Connectivity } \\
\hline & & low & medium & high \\
\hline \multirow{3}{*}{$\begin{array}{c}\text { Ease of link monitoring } \\
\text { and enforcement }\end{array}$} & low & $289,791.43$ & $289,787.24$ & $289,782.32$ \\
\hline & medium & $289,796.09$ & $289,794.93$ & $289,792.01$ \\
\hline & high & $289,800.67$ & $289,800.18$ & $289,799.61$ \\
\hline \multicolumn{5}{|c|}{ Panel C. $50 \%$ risk covered by 1 st bank, $50 \%$ risk covered by 2 nd bank } \\
\hline & & \multicolumn{3}{|c|}{ Connectivity } \\
\hline & & low & medium & high \\
\hline \multirow{3}{*}{$\begin{array}{c}\text { Ease of link monitoring } \\
\text { and enforcement }\end{array}$} & low & $289,681.21$ & $289,677.59$ & $289,674.31$ \\
\hline & medium & $289,683.32$ & $289,681.05$ & $289,680.08$ \\
\hline & high & $289,684.69$ & $289,684.44$ & $289,684.74$ \\
\hline
\end{tabular}

Panel D. $80 \%$ risk covered by 1 st bank, $20 \%$ risk covered by 2 nd bank

\begin{tabular}{clrrr}
\hline & & \multicolumn{3}{c}{ Connectivity } \\
\cline { 3 - 5 } & & low & medium & high \\
\hline & low & $289,566.09$ & $289,565.59$ & $289,565.20$ \\
Ease of link monitoring & medium & $289,567.35$ & $289,566.83$ & $289,566.51$ \\
and enforcement & high & $289,568.51$ & $289,568.35$ & $289,568.03$ \\
\hline
\end{tabular}

Panel E. $100 \%$ risk covered by 1 st bank

\begin{tabular}{clrrr}
\hline & \multicolumn{4}{c}{ Connectivity } \\
\cline { 2 - 5 } & low & low & medium & \multicolumn{1}{c}{ high } \\
\hline \multirow{2}{*}{ Ease of link monitoring } & medium & $289,489.06$ & $289,488.87$ & $289,491.26$ \\
and enforcement & high & $289,490.75$ & $289,488.40$ & $289,488.60$ \\
\hline
\end{tabular}




\section{References}

[1] Franco Modigliani and Merton H. Miller. The cost of capital, corporation finance and the theory of investment. The American Economic Review, 48(3):261-297, 1958.

[2] Brian W. Nocco and René M. Stulz. Enterprise risk management: Theory and practice. Journal of Applied Corporate Finance, 18(4):8-20, 2006.

[3] Sohnke Bartram. Enhancing shareholder value with corporate risk management. Corporate Finance Review, 7(3):8-13, 2002.

[4] Clifford W. Smith and René M. Stulz. The determinants of firms' hedging policies. The Journal of Financial and Quantitative Analysis, 20(4):391-405, 1985.

[5] Deana R. Nance, Jr. Clifford W. Smith, and Charles W. Smithson. On the determinants of corporate hedging. The Journal of Finance, 48(1):267-284, 1993.

[6] Kenneth A. Froot, David S. Scharfstein, and Jeremy C. Stein. Risk management: Coordinating corporate investment and financing policies. The Journal of Finance, 48(5):1629-1658, 1993.

[7] René M. Stulz. Rethinking risk management. Journal of Applied Corporate Finance, 9(3):8-25, 1996.

[8] David Mayers and Jr. Clifford W. Smith. On the corporate demand for insurance. The Journal of Business, 55(2):281-296, 1982.

[9] Peter Tufano. Who manages risk? an empirical examination of risk management practices in the gold mining industry. The Journal of Finance, 51(4):1097-1137, 1996.

[10] Jr. Clifford W. Smith. Corporate risk management: Theory and practice. Journal of Derivatives, 2(4):21-30, 1995.

[11] Dong-Hyun Ahn, Jacob Boudoukh, Matthew Richardson, and Robert F. Whitelaw. Optimal risk management using options. The Journal of Finance, 54(1):359-375, 1999.

[12] D. P. Rutenberg. Maneuvering liquidity assets in a multinational company: Formulation and deterministic solution procedures. Management Science, 16(10):671-684, 1970.

[13] Venkat Srinivasan and Yong H. Kim. Payments netting in international cash management: A network optimization approach. Journal of International Business Studies, 17(2):1-20, 1986. 
[14] John M. Mulvey and Hercules Vladimirou. Stochastic network programming for financial planning problems. Management Science, 38(11):1642-1664, 1992.

[15] Franklin Allen and Douglas Gale. Financial contagion. The Journal of Political Economy, 108(1):1-33, 2000.

[16] C. Kenneth Jones. A network model for foreign exchange arbitrage, hedging and speculation. International Journal of Theoreticl and Applied Finance, 4(6):837-852, 2000.

[17] Jukka Hallikas, Iris Karvonen, Urho Pulkkinen, Veli-Matti Virolainen, and Markku Tuominen. Risk management processes in supplier networks. International Journal of Production Economics, 90(1):47-58, 2004.

[18] Marcel Fafchamps and Flore Gubert. Risk sharing and network formation. American Economic Association, 97(2):75-79, 2007.

[19] Stefan Thurner, Rudolf Hanel, and Stefan Pichler. Risk trading, network topology, and banking regulation. Quantitative Finance, 3(4):306 - 319, 2003.

[20] Michael Boss, Helmut Elsinger, Martin Summer, and Stefan Thurner. Network topology of the interbank market. Quantitative Finance, 4(6):677-684, 2004.

[21] Daniel Egloff, Markus Leippold, and Paolo Vanini. A simple model of credit contagion. Journal of Banking and Finance, 31(8):2475-2492, 2007.

[22] Erlend Nier, Jing Yang, Tanju Yorulmazer, and Amadeo Alentorn. Network models and financial stability. Journal of Economics Dynamics and Control, 31(6):2033-2060, 2007.

[23] Walter Wasserfallen and Christoph Schenk. Portfolio insurance for the small investor in switzerland. Journal of Derivatives, 3(3), 1996.

[24] Pavel A. Stoimenov and Sascha Wilkens. Are structured products fairly priced? an analysis of the german market for equity-linked instruments. Journal of Banking \& Finance, 29(12):2971 $-2993,2005$.

[25] Gianluca Fusai and GIovanna Zanotti. New efficient frontier: Can structured products really improve the risk return profile? Working paper, 2011. 
[26] Darrell Duffie, Nicolae Gârleanu, and Lasse Heje Pedersen. Over-the-counter markets. Econometrica, 73(6):1815-1847, 2005.

[27] Darrell Duffie, Nicolae Gârleanu, and Lasse Heje Pedersen. Valuation in over-the-counter markets. Review of Financial Studies, 20(6):1865-1900, 2007.

[28] Darrell Duffie and David Lando. Term structures of credit spreads with incomplete accounting information. Econometrica, 69(3):633-664, 2001.

[29] Fan Yu. Accounting transparency and the term structure of credit spreads. Journal of Financial Economics, 75(1):53-84, 2005.

[30] Mark Lang and Russel Lundholm. Cross-sectional determinants of analyst ratings of corporate disclosures. Journal of Accounting Research, 31(2):246-271, 1993. 